\title{
Effects of varying trial distribution, intra- and extramaze cues, and amount of reward on proactive interference in the radial maze
}

\author{
J. S. COHEN, S. REID, and K. CHEW \\ University of Windsor, Windsor, Ontario, Canada
}

\begin{abstract}
Rats are typically less accurate in their arm selections in the radial maze over successive trials in a session (Roberts \& Dale, 1981). In the present study, rats' choice accuracy declined when such trials were separated by 2 -min (massed) but not by 2 -h (spaced) intertrial intervals. Changing intramaze visual/tactile arm stimuli (Experiments 1 and 3) or extramaze landmark stimuli (Experiment 4) between trials weakened the massed-trials effect, but changing the number of food pellets per arm, either alone or in conjunction with changes in intramaze cues (Experiments 2 and 3), did not. The rats also tended to avoid the spatial locations of their last four choices on a previous trial during their first four choices on a current trial, and more so with massed than with spaced trials. These findings indicate that intertrial proactive interference (PI) occurred only with massed trials and was weakened by changing intra- and extramaze cues between such trials.
\end{abstract}

The radial maze task was developed to investigate rats' spatial working memory (Olton, 1978, 1979; Olton, Collison, \& Werz, 1977; Olton \& Samuelson, 1976). In this task, a rat collects rewards at the end of arms that radiate from a central choice platform. Entered arms are not rebaited during a trial, and, consequently, rats quickly learn to avoid previously selected arms. Indeed, rats only occasionally reenter earlier sampled arms during their final choices in a trial. Olton (1978) also noted that, over repeated trials, rats maintained accurate performance on their initial choices when those trials were separated by 1-min intertrial intervals (ITIs). Therefore, he suggested that rats are immune from intertrial proactive interference (PI) because they apparently "reset" their working memory for arm locations at the end of each trial.

Roberts and Dale (1981) later questioned this "reset" hypothesis, noting that the rats in Olton's $(1978,1979)$ experiments as well as their own were more likely to reenter arms during their final choices on trials occurring later in a session. Increasing ITIs from 60 to $240 \mathrm{sec}$ did not affect this deterioration of choice accuracy. Their rats also tended to avoid the arm locations they had visited on the last four choices of the previous trial during their

The present study is based on research conducted by the second author in partial fulfillment for her B.A. Honours degree at the University of Windsor. Funds from the Research Board of the University of Windsor to the senior author supported this research. Portions of this study were presented at the meeting of the Canadian Society for Brain, Behaviour, and Cognitive Science, at Laval University, Quebec, June 1992. The authors thank G. Namikas at the University of Windsor for his thoughtful criticisms of the paper. Correspondence concerning this article should be addressed to J. S. Cohen, University of Windsor, Department of Psychology, Windsor, ON, Canada N9B 3P4. initial four choices of a current trial. This tendency to avoid the final arm locations from a prior trial is further evidence against an end-of-trial "resetting" process in spatial working memory. Dale and Roberts (1986) replicated these effects when arms were baited with water as well as with food pellets.

Roberts and Dale (1981) proposed that massing trials causes rats to become less certain about whether they last visited an arm during the current or previous trial. Such confusions occur because massing trials reduces the difference between the time an arm might have been visited in the previous versus the current trial. This temporal discrimination should be easier when trials are more widely spaced. This account is similar to temporal discrimination (D'Amato, 1973) and trial distinctiveness (Santiago \& Wright, 1984; Wright, Santiago, \& Sands, 1984; Wright, Urcuioli, \& Sands, 1986) explanations of PI in nonspatial, delayed matching performance. According to the trial distinctiveness account, however, animals can acquire nontemporal means to determine the recency of trial events. For example, pigeons and monkeys do not show intertrial PI in nonspatial, successive delayed matchingto-sample and serial-probe recognition tasks that involve trial-unique stimuli (Santiago \& Wright, 1984; Wright et al., 1984).

The present study consisted of four experiments that were designed to test this recency confusion model of intertrial PI in the radial maze. In the first two experiments, rats received repeated trials separated by $2 \mathrm{~h}$ (spaced trials) or 2 min (massed trials) in a radial maze. All trials were massed in the last two experiments. The rats were also exposed to changes in intramaze visual/ tactile arm stimuli (Experiments 1 and 3), extramaze landmark cues (Experiment 4), and number of food pellets 
per arm (Experiments 2 and 3) between trials. According to the recency confusion hypothesis, the rats were expected to show a greater decline in choice accuracy over massed than over spaced trials. If rats can also use nontemporal cues to determine when they last visited an arm, we predicted that the decline in accuracy over massed trials would be less when trials were differentiated by intra- or extramaze cues or by the amount of food in each arm.

\section{EXPERIMENT 1}

The first experiment was designed to determine the effects on radial maze performance of varying the distribution of trials and of the visual/tactile arm stimuli used on successive trials. Three different types of arm stimuli were used: wire-mesh (gridded) arms, black, smooth arms, and gray arms with yellow plastic pegs. These were chosen partly on the basis of prior research from our laboratory (Cohen, Burkhart, Jones, \& Innis, 1990), which showed that rats can distinguish between smooth and gridded arms within a trial. Other researchers (Chamizo \& Mackintosh, 1989; Chamizo, Sterio, \& Mackintosh, 1985; Kraemer, Gilbert, \& Innis, 1983) have also reported that rats perform accurately with similarly differentiated intramaze stimuli (smooth or rubber vs. sandpaper floors).

Three groups of rats received alternating three-trial sessions in which trials were either massed or spaced. The rats in the same-arm-cue group were exposed to only one type of arm cue on the three trials of a session, although this cue was varied between sessions. The rats in the constant-sequence group received different arm cues over the successive trials in each session, but the sequence of cues was constant from session to session. Those in the varied-sequence group also received different arm cues over successive trials, but the sequence of these cues was varied between sessions as well. We expected that the rats in all three groups would be less accurate in their choices over massed than over spaced trials. Additionally, the same-arm-cue rats were expected to display a greater massed-trials effect than those in the other two groups. For the latter groups, varying arm cues over trials was expected to reduce confusion about when those arms were last visited. Finally, if rats can learn to associate each arm cue with a particular trial, the constant-sequence group might be least disrupted by massing trials.

\section{Method}

Subjects. The subjects were 18 naive, male Long-Evans hooded rats purchased from Charles River Breeding Farms, St. Constant, Quebec. They were 105 days old and weighed at least $300 \mathrm{~g}$ at the start of the experiment. The rats were housed in individual holding cages during the experimental session and in large group cages ( 3 per cage) between sessions. The colony room was maintained on a 12:12-h dark:light cycle; the rats were run during the dark portion of this cycle. Each rat was reduced to $85 \%$ of its free-feeding weight, adjusted to the growth curve for this strain. Each rat received 15-20 $\mathrm{g}$ of dry food (Purina Rodent Chow) in its individual holding cage about $1 \mathrm{~h}$ after its experimental session.

Apparatus. The apparatus was an elevated radial maze, constructed of aluminum and plastic materials. It stood $70 \mathrm{~cm}$ above the floor in the experimental room and consisted of eight identical arms, spaced $45^{\circ}$ apart, radiating outward from a central, octagonal choice area. The octagonal choice area was $51 \mathrm{~cm}$ wide, was painted flat black, and was surrounded by a $43-\mathrm{cm}$-high enclosure consisting of eight $13-\mathrm{cm}$-wide guillotine doors. Each arm was made from 10-cm-wide $\times 5$-cm-deep channel aluminum, and each extended $61 \mathrm{~cm}$ from the choice platform. A $2.5-\mathrm{cm}$-diam food cup was placed at the end of each arm. The arms were attached to the central platform in such a way that metal inserts in the arms were level with the choice area's floor and the food cups. There were three types of arm inserts: solid black, solid gray with diagonally crossing 2.5-cm-high yellow plastic pegs (Muret electrical connectors), and gridded mesh of No. 2 galvanized hardware cloth. Clear, 40$\mathrm{cm}$-high $\times 30$-cm-long Plexiglas barriers were located between the arms to prevent the rats from jumping across adjacent arms.

The maze was enclosed in a 295-cm-wide octagonal chamber made of $180-\mathrm{cm}$-high $\times 122-\mathrm{cm}$-wide wooden panels. Each arm of the maze radiated toward the edge of two adjacent wall panels of the enclosure. The north, east, south, and west panels contained extramaze stimuli during training; the northeast, southeast, southwest, and northwest panels were bare. Half of the northeast panel was hinged like a door to allow the experimenter to enter and leave the maze area. The north and south panels contained a series of small, clear Christmas tree lights (Alderbrook Sparkle Lites, London, ON) arranged in a serpentine fashion. The lights on the south panel flashed on and off approximately every $4 \mathrm{sec}$, while those on the north panel remained on. Black wrapping paper with silver streaks covered the west panel. Plastic and dried flowers were attached in a random fashion to the east panel. The room was moderately illuminated by four recessed $150-\mathrm{W}$ incandescent ceiling lights. A video camera suspended $80 \mathrm{~cm}$ above the center of the maze provided a complete image of the maze on a monitor outside the enclosure. The monitor was located near the north wall of the room in an area that also contained controls for the guillotine doors. The experimenter remained out of the rat's sight at this observing and control station.

Procedure. All of the rats received a preexperimental and an experimental training phase. Preexperimental training consisted of 5 sessions of arm-entry shaping, followed by 12 sessions of arm-cue presentation. No extramaze stimuli were present during the first stage. Each food cup was baited with two 45-mg dustless food pellets (BioServe, Frenchtown, NJ). The experimenter stood by the two black arms radiating to the left and right of the bare northeast wall panel and trained the rats to approach and eat from the food cups. Initially, the rats were placed directly on each arm. Later, they were placed in the central choice area and shaped to run to either arm when its guillotine door was raised.

Extramaze stimuli were present during the second stage of preexperimental training. The rats received one trial per day for 12 days. The experimenter remained at the observing and control station while the rat sampled all the arms that contained one type of insert (black, gridded, or pegged) during a session. Each arm cue occurred on four sessions in random order, with the restriction that none appeared on more than two successive sessions. On each trial, a rat was permitted 12 choices within 10 min to enter all arms. The experimenter began a trial by placing a rat in the choice area and then raising all guillotine doors while at the observing and control station. When the rat entered an arm, the experimenter lowered all doors except the door to the entered arm, which was lowered when the rat reentered the choice area. The rat was then confined in the choice area for $15 \mathrm{sec}$ before the experimenter raised all the doors for its next choice. The 15 -sec interchoice delay was used to prevent the rats from developing stereotypic choice sequences (Olton et al., 1977). After the rat had visited the last arm, the experimenter removed it from that arm, replaced it in its holding cage, and dampmopped the maze with a weak vinegar solution.

In the experimental training phase, the rats received three trials per session for 48 sessions. Odd-numbered sessions contained massed 
trials that were separated by 2 -min ITIs. Even-numbered sessions contained spaced trials that were separated by 2 -h ITIs. A rat remained in its holding cage between trials. The rats were randomly divided into three equal groups for this phase. For the same-armcue group, one arm insert was used in all arms for all three trials of a session, with a different arm cue occurring over sessions. These cues were arranged on a random and equal basis within each trial distribution condition, with the restriction that the same cue could not occur on more than two successive sessions. For the other two groups, arm cues were changed over the three successive trials in each session. Six different sequences of arm cues were possible: Gridded-Pegged-Black (G-P-B), B-G-P, P-G-B, B-P-G, G-B-P, or $\mathrm{P}-\mathrm{B}-\mathrm{G}$. These sequences were counterbalanced in the constantsequence group so that each rat received the same sequence on every session. By contrast, each rat in the varied-sequence group received each sequence equally often on massed- and spaced-trial sessions in a random order, with the restriction that none could occur on more than two consecutive sessions.

\section{Results and Discussion}

Maze performances, as measured by choice accuracy on each trial and by overlap scores between trials, were analyzed in the experimental phase as follows. A rat's choice accuracy score on each trial was the ratio of its rewarded choices to the total number of choices (no more than 12) required to sample all arms. By the end of preexperimental training, the rats seldom, if ever, failed to visit all the arms within the 12 choices. Consequently, choice accuracy scores ranged from .67 (when a rat required 12 choices; i.e., made four reentries to sample all arms) to 1.00 (when it required only 8 choices; i.e., made no reentries to sample all arms). A rat's overlap score was the proportion of arm locations, from its last four different choices on a prior trial, that it entered on its first four choices of a current trial. If the initial choices on a trial are unrelated to the final choices on a previous trial, an average of two overlapping choices or an overlap score of .50 would be expected. This measure is similar to that used by Roberts and Dale (1981).

Choice accuracy. The rats seldom, if ever, reentered arms before their sixth choice. Furthermore, choice accuracy did not systematically change over sessions. Figure 1 shows the choice accuracy for each group by trial in the massed and spaced conditions. A three-way analysis of variance (ANOVA; groups $\times$ trial distribution $\times$ trial, with repeated measures on the last two factors) revealed significant interactions between trial distribution and trial $[F(2,30)=15.66, p<.001]$ and between all three factors $[F(4,30)=3.64, p<.05]$. Post hoc tests (Newman-Keuls, $\alpha=.05$ ) supported the observation from Figure 1 that all the rats showed a significant decrease in accuracy on the second and third trials of the massed condition relative to the first trial, and to levels significantly below those in the spaced condition. The slight changes in accuracy over trials in the spaced condition were not significant. Also, the rats did not differ in their accuracy of arm selections on the first trial in the spacedversus massed-trials sessions. Finally, the same-arm-cue rats showed a steeper decline in accuracy over massed trials than those in the other two groups, although this

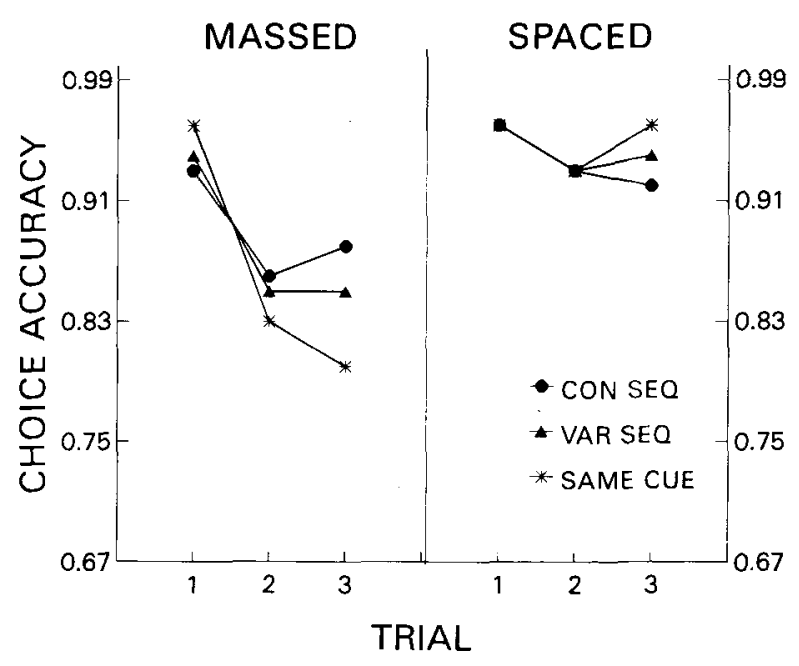

Figure 1. Mean choice accuracy for each group on each trial during sessions with massed and spaced trials in Experiment 1. CON SEQ = constant sequence of changes in arm cues; VAR SEQ = varied sequence of changes in arm cues; SAME CUE = same arm cue over all trials in a session.

effect was only significant on the third massed trial. No significant differences were found between the constantand varied-sequence groups.

Overlap scores. Overlap scores for Trials 2 and 3 were each analyzed by separate two-way ANOVAs (groups $x$ trial distribution). Neither analysis uncovered significant effects due to groups; however, an ANOVA showed a significantly lower proportion of overlapping choices on Trial 2 in the massed- than in the spaced-trials condition $[.42$ vs. $.48 ; F(1,15)=6.83, p<.05]$. Furthermore, the rats overlapped their choices significantly less than chance $(.50)$ only within the massed-trials condition. The comparison between massed and spaced trials for Trial 3 produced a similar but nonsignificant difference [. $45 \mathrm{vs}$. $.48 ; F(1,15)=2.29, p>.10]$. The rats still overlapped their choices significantly less than chance, but only within the massed-trials condition.

The results from this experiment indicate that spacing trials $2 \mathrm{~h}$ apart within a session produced very little, if any, PI from preceding trials in the session, whereas changing intramaze cues weakened, but did not eliminate, the more substantial PI observed over massed trials. Changing arm cues between trials in a constant sequence did not reduce PI any more than changing those cues in a varied fashion, however. Overlap scores below chance were also evident only in the massed-trials condition. Therefore, the rats did not completely reset their working memory for arm locations over 2 -min ITIs, but appeared to do so over 2-h ITIs. This tendency to avoid entered arms from an immediately preceding trial indicates that the rats did not completely differentiate the current from the prior trial. This incomplete differentiation might have led to confusions about when some arms had been entered during the final choices of an ongoing trial, thus leading to the drop in accuracy on the later trials. 


\section{EXPERIMENT 2}

The purpose of Experiment 2 was to determine if trialby-trial changes in the number of food pellets per arm might also attenuate intertrial PI in the same way that the changes in arm cues did in Experiment 1. To this end, rats in three separate groups received either the same number of pellets per arm on all trials (three-pellets group), a consistent increase or decrease in the number of pellets over trials (constant-sequence group), or variations in the change in number of pellets over trials (varied-sequence group). In this experiment, all the arms remained black on all trials.

We expected that the rats would continue to display more accurate arm selections in the spaced- than in the massed-trials condition, but that the rats exposed to changes in the number of pellets per arm over trials, especially those in the constant-sequence group, would be less disrupted by the massing of trials than the rats that always received three pellets per arm on every trial. The latter prediction was based on data from Hulse (1978), who showed that rats learn to accurately anticipate changes in reward magnitude (from 14 to 7 to 0 pellets) in the straight-alley runway when such changes occur in a monotonic rather than a nonmonotonic fashion. We thought that our rats might similarly learn to anticipate reward magnitudes that either always monotonically decreased between trials, from 5 pellets per arm ( 40 pellets per trial) to 3 pellets per arm ( 24 pellets per trial) and then to 1 pellet per arm ( 8 pellets per trial), or monotonically increased in the opposite order. If so, these constant changes in reward magnitude might reduce the massed-trials effect in the constant-sequence group relative to that in the varied-sequence and three-pellets groups.

\section{Method}

Subjects and Apparatus. The same rats, apparatus, basic design, and procedures of the first experiment were used, except that the arms remained black over all trials, and the number of food pellets per arm was varied over trials for some of the rats. As in Experiment 1, massed and spaced trials continued to alternate across sessions. The rats were reassigned to three groups of 6 subjects each in this experiment so that one third of the subjects from each group in Experiment 1 were represented in each of the current groups. One group of rats always received three pellets per arm on each trial (three-pellets group). The other two groups received one or five pellets per arm on the first trial, three pellets per arm on the second trial, and one or five pellets per arm on the third trial of each session. The varied-sequence group received both the ascending (1-3-5) and descending (5-3-1) changes in the number of pellets in a random order over sessions, with the restriction that the same order not occur on more than two sessions in a row. For the constant-sequence group, half always received the ascending order of pellets per arm (1-3-5) over trials, and the other half always received the descending order (5-3-1).

\section{Results and Discussion}

Choice accuracy. Figure 2 shows the choice accuracy for each group on each trial in the massed and spaced conditions. The only detectable and consistent difference occurred as a function of trial distribution: The rats showed

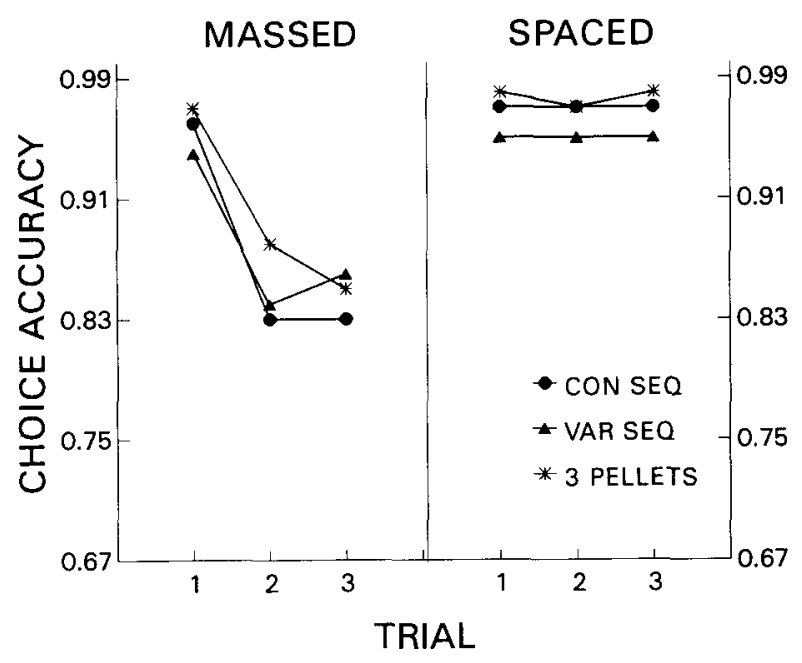

Figure 2. Mean choice accuracy for each group on each trial during sessions with massed and spaced trials in Experiment 2 . CON SEQ = constant sequence of changes in number of pellets per arm; VAR SEQ = varied sequence of changes in number of pellets per arm; 3 PELLETS = three pellets per arm on each trial.

a decrease in choice accuracy over massed trials only. No consistent differences were observed over spaced trials or between groups. These observations were supported by a significant interaction between trial distribution and trial $[F(2,30)=39.84, p<.01]$. Post hoc tests revealed that the rats' accuracy significantly declined to similar levels on the second and third trials in the massed condition, and that this level was significantly lower than that on the second and third spaced trials. As in Experiment 1, the rats did not differ in accuracy on the first trial in the massed- versus the spaced-trials sessions.

Overlap scores. Statistical analyses revealed that the rats displayed significantly lower overlap scores on Trials 2 and 3 in the massed- than in the spaced-trials condition [Trial $2, .42$ vs. $.46, F(1,15)=7.59, p<.05$; Trial $3, .41$ vs. $.44, F(1,15)=5.86, p<.05]$. Overlap scores in both conditions were significantly below chance, however.

In summary, changing the number of pellets per arm over trials had no discernible effect on performance. However, the effects of trial distribution found in Experiment 1 were replicated here; that is, the rats showed poorer accuracy over trials only in the massed-trials condition, and, again, there was less overlap in choices over massed than over spaced trials. The rats also showed below-chance overlap scores in the spaced-trials condition, however, and so did not appear to completely "reset" their working memory for arm locations as they seemed to do in the spaced-trials condition of Experiment 1.

\section{EXPERIMENT 3}

The first two experiments showed that changing intramaze arm cues between trials, but not the number of pellets per arm (or total amount of food in the maze), at- 
tenuates PI over massed trials. Further, the rats that always experienced changes in arm cues between the trials within a session showed slightly, but reliably lower PI over massed trials than those that always received the same type of arm cue. It is noteworthy that changing arm cues within a session did not eliminate PI over massed trials and that the attenuation of PI was a between-group effect. The major purpose of Experiment 3 was to see if varying arm cues over trials would diminish or even eliminate intertrial PI in a within-subjects design. Such an effect would provide stronger evidence for the recency confusion hypothesis of PI in rats' spatial memory. A second, but no less important goal of this experiment was to attempt to increase the effectiveness of arm-cue changes between trials in order to eliminate intertrial PI.

Changing the intramaze arm cues between trials may not have eliminated PI in Experiment 1 because rats typically attend more to distal, extramaze landmark cues (Chamizo et al., 1985; Kraemer et al., 1983). Nevertheless, increasing the salience of proximal arm cues might promote greater attention to them and thus further reduce the massed-trials effect. In Experiment 3, we attempted to increase the salience of the proximal arm cues by pairing each with a specific number of food pellets. Rats received only massed trials in this experiment, which consisted of two phases. In the first phase, arm cues and their associated number of pellets per arm were changed over the three trials in each session. This phase was run to determine whether the massed-trials effect would disappear with such extended training. The second phase tested whether the rats would show sensitivity to same versus different arm cues between trials in a session. This phase consisted of alternating sessions with trials containing different arm cues (as in the first phase) versus trials containing the same arm cue. The rats were expected to display a greater massed-trials effect on sessions with samethan with different-arm-cue trials.

\footnotetext{
Method

Subjects and Apparatus. The 6 rats from the three-pellets group in Experiment 2 were used in this experiment. The other rats were excluded because they had experienced different numbers of pellets over trials. The three types of arm inserts used in Experiment 1 were used in this experiment as well.

Procedure. All the rats received three massed trials per session for 54 sessions. The experiment was divided into two phases. The first consisted of 18 sessions in which each trial involved a different arm cue and its associated number of pellets. The second consisted of $\mathbf{3 6}$ sessions that alternated between those in which all trials involved the same arm cue and its associated number of pellets (oddnumbered sessions) and those involving trials with different arm cues (even-numbered sessions). Black arms continued to be baited with three pellets for all the rats, whereas gridded arms and pegged arms were baited with one and five pellets, respectively, for 3 rats and five and one pellets, respectively, for the other 3 rats. The various possible sequences of arm cues occurred equally often in a random order over the first 18 sessions and over the 18 evennumbered sessions of the second phase, as they had in the variedsequence group in Experiment 1. The 6 sessions consisting of only black, only pegged, or only gridded arms on each trial were randomly and evenly distributed over the odd-numbered sessions.
}

\section{Results and Discussion}

Choice accuracy. Data from the first phase were partitioned into three blocks of six sessions each to assess any changes in the massed-trials effect over extended training. Figure 3 shows mean accuracy scores on each trial during the first, second, and final block of six sessions. As seen in this figure and supported by a significant effect for trials $[F(2,10)=9.85, p<.01]$, the rats showed slightly poorer choice accuracy on the second and third trials in each block relative to the first trial. This massed-trials effect was clearly weaker than that seen for the three-pellets group in Experiment 2 (see Figure 2), but did not disappear as expected. The gradients did not differ significantly over blocks.

Figure 4 shows mean accuracy scores during the alternating sessions of same and different arm cues in the second phase. These data were partitioned into two blocks of 18 sessions each and were analyzed by a three-way ANOVA (block $\times$ type of session $\times$ trial). As seen in this figure and confirmed by a main effect for trial $[F(2,10)=$ $23.73, p<.001]$, the rats continued to show lower overall accuracy on the second and third trials than on the first. The type of session had an influence on performance only within the first 18 sessions, however. Within that block, the rats showed a greater drop in accuracy over the trials with the same than with different arm cues. During the second block of 18 sessions, their performance on trials with the same arm cue improved to the level of that observed on trials with different arm cues. The interaction between block and type of session to confirm this observation barely missed significance $[F(1,5)=5.98, p=$ .059 ], but a significant main effect for type of session

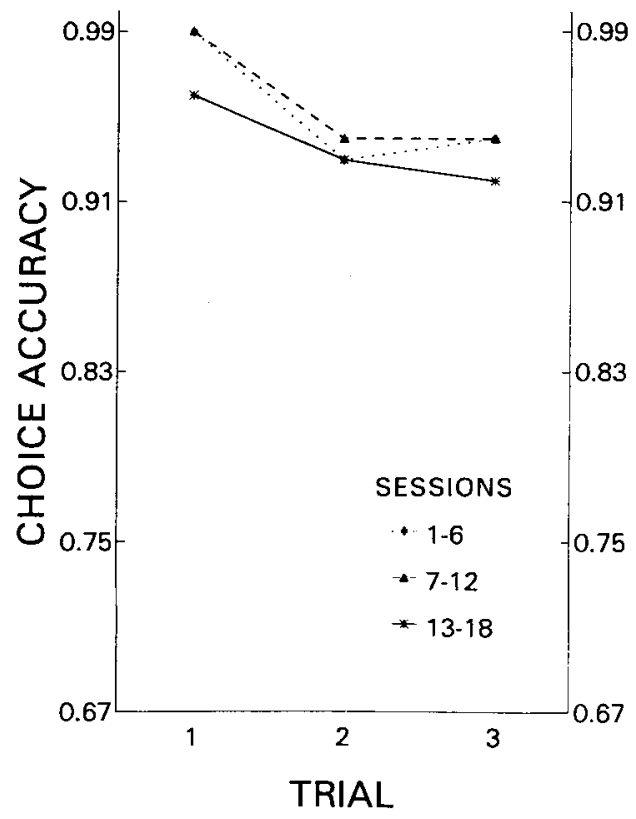

Figure 3. Mean choice accuracy on each trial over the first 18 sessions of training partitioned into blocks of 6 sessions in Experiment 3. All trials were massed, and arm cue and its associated number of pellets per arm differed between trials. 


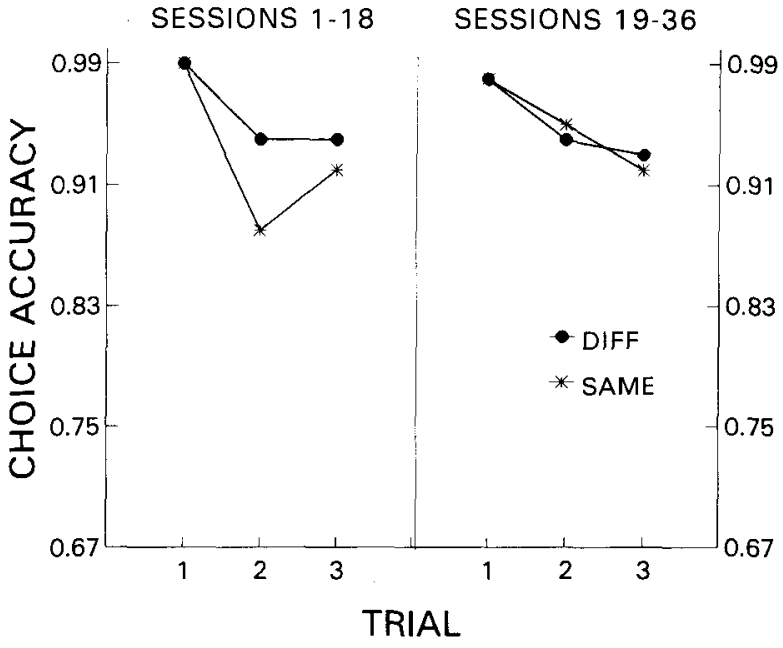

Figure 4. Mean choice accuracy on each trial during the first and second block of 18 sessions of testing in Experiment 3. All trials were massed, and sessions alternated between those in which arm cues and their associated number of pellets per arm differed (DIFF) or were the same (SAME) between trials of a session.

(pooled over blocks) was found $[F(1,5)=49.00, p=$ $.001]$. When a separate two-way ANOVA (type of session $\times$ trial) was carried out for each block of sessions, though, a significant interaction was found only for the first block $[F(2,10)=15.28, p<.01]$.

A subsidiary two-way ANOVA (number of pellets $x$ trial) on the data from sessions with trials containing the same arm cue assessed the possible effect of different amounts of food per arm on choice accuracy over sessions. Number of pellets per arm did not significantly affect the rats' performance as either a main $[F(2,10)=$ $2.72, p>.10]$ or interactive factor $[F(4,20)=1.74$, $p>.10$ ]. Thus, varying the amount of pellets per arm within (i.e., on even-numbered) sessions or between (i.e., on odd-numbered) sessions did not affect choice accuracy. This finding replicates earlier research showing that the size of the food reward does not affect rats' performance in the radial maze (Gaffan, Hansel, \& Smith, 1983; Olton \& Samuelson, 1976).

Overlap scores. Session type did not significantly affect the degree to which locations of the first four choices on Trials 2 or 3 were determined by the locations of the last four choices from the preceding trial $[F \mathrm{~s}(1,5)=.44$ and $.006, p s>.50]$. On each type of session, the rats averaged an overlap score of .44-a score significantly below . 50 .

These results show that pairing each arm cue with a specific number of pellets did not increase rats' attention to changes in these cues enough to eliminate the massedtrials effect. Interspersing sessions with trials involving the same arm cue in the second phase disrupted performance, but the effect was transitory, disappearing on the second block of 18 sessions. This temporary effect may only reflect the novelty of unexpected trials with the same arm cue following extensive training, in which arm cues were changed between trials in each session.

Together, the results from Experiments 1, 2, and 3 suggest that manipulating arm cues, but not the amount of food per arm over trials, affects intertrial PI. Before dismissing the possibility of any effect of changes in the amount of food per arm on PI, however, one should note that relatively small changes in the amount of food per arm were used (1 vs. 3 vs. 5 pellets). Such small differences may not have been noticed by or may not have differentially motivated our subjects. Research on successive contrast in rats has shown that a larger change in reward size (between 1 and 12 pellets) causes sudden postshift increases or decreases in running speed in the straight alley (Franchina \& Brown, 1971). Perhaps larger differences in the amount of reward might also have attenuated PI in our task.

\section{EXPERIMENT 4}

The results from Experiments 1 and 3 showed that changing the intramaze arm cues between the successive trials in the massed-trials condition attenuates, but does not eliminate, intertrial PI. Perhaps intertrial PI could be reduced even more if the relatively salient distal landmark cues are changed between trials. To test this prediction, we ran another within-subjects experiment in which distal landmark cues were sometimes varied and sometimes kept the same between two massed trials in a session.

\section{Method}

Subjects and Apparatus. Nine rats were selected by randomly choosing 3 rats from each of the three groups in Experiment 2 . In the present experiment, each arm of the maze was black and was baited with three pellets. The north and south panels of the octagonal enclosure contained the steady and flashing Christmas tree lights, respectively, as in the previous experiments, and they served as one set of landmark cues (SL/FL). The east and west panels contained a permanent $1 \mathrm{~m}^{2}$ black square instead of the original stimuli. A second set of landmark cues was created by placing a removable 47-cm-diam white circle in the middle of the black square on the west panel; the black square on the east panel remained bare (BW/B). An extendable workshop lamp with a 40-W incandescent light bulb (Luxolamp, Montreal, Quebec) was also placed over the east and west panels to illuminate these new landmark cues.

Procedure. All the rats received two massed trials per session for 48 sessions. In addition, two kinds of trials were presented. On an SL/FL trial, the steady and flashing Christmas tree lights on the north and south panels were presented, while both the east and west panels' black squares were bare and not illuminated. On a BW/B trial, the black squares on the east and west panels were illuminated, the west panel's black square contained the white circle, and the Christmas tree lights were unlit. These trials could occur in four possible two-trial sequences; two involved the same cues (SL/FL-SL/FL and BW/B-BW/B), and the other two involved different cues (SL/FL-BW/B and BW/B-SL/FL). Every rat experienced each sequence equally often in a random order, with the restriction that no particular sequence or type of sequence (same or different) could occur on two consecutive sessions.

The experimenter changed wall panel stimuli and turned the workshop lamps and Christmas tree lights on or off (or went through the motions of doing so) while a rat waited in its holding cage be- 
tween trials. Thus, the rats experienced equivalent ITIs of approximately 2 min between same- and different-landmark trials.

\section{Results and Discussion}

The 48 sessions were divided into two blocks of 24 sessions, and the data were compared between these blocks. The first 24 sessions served as an initial training block for the introduction of a new set of landmarks (BW/B) and for the new procedures of presenting each set of landmarks.

Choice accuracy. Figure 5 shows mean choice accuracy for each sequence of massed trials for the first and second block of sessions. A four-way ANOVA (Trial 1 cues $\times$ Trial 2 cues $\times$ trial $\times$ block) revealed a significant effect of trial $[F(1,8)=28.67, p<.01]$ and a significant interaction among all four factors $[F(1,8)=$ $32.58, p<.01]$. As can be seen in this figure, the rats showed poorer choice accuracy on the second trial within most sequences in each block of sessions. However, they showed very little decline in choice accuracy across trials within the BW/B-SL/FL sequence in the second block of sessions. Separate three-way ANOVAs on the data from each block of sessions supported this observation by showing a significant interaction among the three factors (Trial 1 cues $\times$ Trial 2 cues $\times$ trial) only in the second block of sessions $[F(1,8)=10.71, p<.05]$. This effect resulted from significantly lower choice accuracy on the second than on the first trial for all but the $\mathrm{BW} / \mathrm{B}-\mathrm{SL} / \mathrm{FL}$ sequence.

Overlap scores. The rats tended to avoid their last four chosen arm locations on Trial 1 during their first four choices on Trial 2. A three-way ANOVA (Trial 1 cues $\times$ Trial 2 cues $\times$ block) revealed that they showed a slight but significant increase in overlap scores, from .38 in the

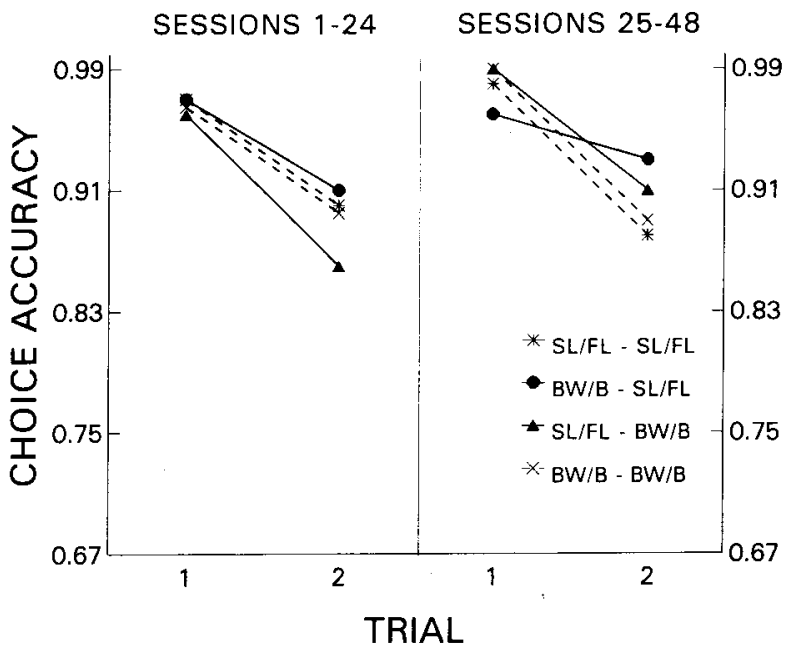

Figure 5. Mean choice accuracy at each trial during the first and second block of 24 sessions in Experiment 4 . All trials were massed, and extramaze wall panel landmark cues were either the same or differed between the two trials of a session in the following sequences: steady lights/flashing lights (SL/FL)-SL/FL; black square with white circle/black square (BW/B)-BW/B; SL/FL-BW/B; BW/B-SL/FL. first block to .43 in the second block $[F(1,8)=6.83, p<$ .05]. A significant interaction between Trial 1 and Trial 2 cues was also found $[F(1,8)=11.04, p<.05]$. This interaction resulted from the rats' showing less overlap in their Trial 2 choices in the BW/B-SL/FL than in the SL/FL-SL/FL sequence (.36 vs. .44). There was no significant difference in Trial 2 overlap scores between the SL/FL-BW/B and BW/B-BW/B sequences (.42 vs. .40). All overlap scores remained significantly below chance, however.

The results from this experiment indicate that changing extramaze landmark cues between trials does not uniformly reduce PI. The massed-trials effect was eventually eliminated only within the BW/B-SL/FL sequence, but the rats still avoided arm locations visited at the end of their first trial somewhat more within that sequence. These latter results were surprising, because the elimination of intertrial PI within the BW/B-SL/FL sequence should have been accompanied by greater overlap scores approaching chance.

\section{GENERAL DISCUSSION}

The findings of this study appear to support the recency confusion account of PI (Roberts \& Dale, 1981), which proposes that repeating trials reduces rats' ability to determine whether an arm was last visited during the current or previous trial. Demarcating trials by intramaze arm cues or distal landmark cues reduced this confusion; increasing ITI duration to $2 \mathrm{~h}$ appeared to eliminate it. The results from Experiments 2 and 3 indicate that the rats either did not or could not demarcate trials on the basis of the amounts of food used in these experiments.

It is noteworthy that in Experiment 3, changing arm cues between trials produced only a transient attenuation of the massed-trials effect. However, this seems largely attributable to the fact that the subjects improved their performance over sessions with massed trials containing the same arm cue. In addition, the same subjects were run in all experiments and so tended to show a general improvement in their performance over successive experiments. As a result, intertrial PI seemed to dissipate across experiments. This is particularly evident for the 6 rats in Experiment 3 that comprised the three-pellets group in Experiment 2 (compare massed-trials panel of Figure 2 with Figures 3 and 4). Hoffman and Maki (1986) also found reliable intratrial PI only in the first of their two experiments using the same rats. Perhaps extended training allowed our rats to improve their ability to temporally differentiate between trials separated only by 2 -min ITIs and so reduced recency confusions. Roberts and Dale (1981), however, did not see a similar effect over successive trials when they increased ITIs to $240 \mathrm{sec}$, although their rats did not receive the extensive training that ours did. It should also be noted that the general dissipation of intertrial PI from extended training in our rats may have also obscured the expected effects of changing the distal landmark cues in Experiment 4 . Changing those cues produced only a small and inconsistent increase in accuracy. 
The overlap scores in this study replicate results from earlier research (Roberts \& Dale, 1981) showing that rats do not automatically reset their working memory at the end of a trial. Like the intertrial agreement effect in nonspatial delayed matching-to-sample (DMTS) with pigeons (Edhouse \& White, 1988), the overlap effect may be considered a direct measure of intertrial PI. The intertrial agreement effect refers to the fact that pigeons are more likely to err in selecting a comparison stimulus in the current trial if the sample (and correct comparison stimulus selection) from the immediately previous trial are opposite to that on the present one. Edhouse and White dismissed a recency confusion account of the disruption from massing trials in DMTS tasks because the intertrial agreement effect was unrelated to trial distribution. The present study, however, showed that the rats' tendency to avoid their last entered arm locations from a previous trial during their initial choices of the current trial was inversely related to intertrial interval. In other words, overlap scores were lower between massed trials than spaced trials in both Experiments 1 and 2. This relationship is in accordance with the recency confusion notion of intertrial PI. Perhaps the smaller variations in ITIs typically used in DMTS tasks (e.g., compare 5-sec, 12-sec, and 20-sec ITIs in Edhouse \& White's study with the 2-min and 2-h ITIs in the present study) obscured a relationship between that factor and the intertrial agreement effect.

Methods other than changing intra- and extramaze cues between trials may also reduce recency confusions across massed trials. Dallal and Meck (1990), for example, reported that rats were less disnupted over massed trials in a 12-arm maze when they experienced different types of food (sunflower seeds, pellets, puffed cereal) within each trial at fixed than at varied arm locations across trials. Rats exposed to fixed food-type locations tended to select arms containing puffed cereal as their final choices in each trial (Dallal, personal communication, November 1992). Consequently, recency confusions could have been reduced if the animals had recognized that the arm locations containing the least-preferred food were most likely to have been visited on a previous trial rather than during the initial choices on the present one. It is noteworthy that this manipulation, too, did not eliminate the massed-trials effect. Perhaps varying the number of food pellets per arm at fixed locations within each trial (rather than over trials) might have attenuated the massed-trials effect in the present study. Changing the quality (and preference) of food rather than the amount (either alone or in conjunction with associated arm cues) over trials might also have had that effect. Certainly, other evidence for the recency confusion account of intertrial PI rests upon discovering other, more potent ways of demarcating trials.

Finally, we note that although spacing trials $2 \mathrm{~h}$ apart eliminated PI in the present study, other researchers report PI across even more widely spaced intervals (up to 5 h; see Hoffman \& Maki, 1986; Maki, Beatty, \& Clouse, 1984; Maki, Hoffman, \& Fritsche, 1986). Unlike the present study, researchers in Maki's laboratory used a seg- mented, within-trial procedure in which rats were first forced into half of the arms of the maze in a "study" segment of the trial. After a delay of about $1 \mathrm{~h}$, they received a free-choice, "test" segment in which they could have collected the remaining food by choosing the previously blocked arms. Presenting an "interference" segment, in which they were forced to some or all of the arms immediately prior to the study segment, reduced their accuracy during the final, free-choice test segment. Such "intratrial" PI was particularly noticeable when the rats were forced to arm locations during the interference segment opposite those during the study segment. Repeating this type of double-segmented trial up to $5 \mathrm{~h}$ later also generated poorer free-choice accuracy or intertrial PI. Perhaps the rats from Maki's laboratory displayed more persistent intertrial PI because they tried to retain arm locations over long delays between trials as they had over long delays within a trial (between study and test segments). Our rats might not have been as prone to retain such potentially interfering information over long ITIs because they did not have to retain visited arm locations over long delays within a trial.

\section{REFERENCES}

Chamizo, V. D., \& Mackintosh, N. J. (1989). Latent learning and latent inhibition in maze discriminations. Quarterly Joumal of Experimental Psychology, 41B, 21-31.

Chamizo, V. D., Sterio, D., \& Mackintosh, N. J. (1985). Blocking and overshadowing between intra-maze and extra-maze cues: $A$ test of independence of locale and guidance learning. Quarterly Journal of Experimental Psychology, 37B, 235-253.

Cohen, J. S., Burkhart, P., Jones, N., InNis, N. K. (1990). The effects of an intramaze cue search rule on rats' spatial working memory. Behavioral Processes, 22, 73-88.

DALE, R. H. I., RobeRTS, W. A. (1986). Variations in radial maze performance under different levels of food and water deprivation. $A n$ imal Learning \& Behavior, 14, 60-64.

DALLAL, N. L., \& MECK, W. H. (1990). Hierarchical structures: Chunking by food type facilitates spatial memory. Journal of Experimental Psychology: Animal Behavior Processes, 16, 69-84.

D'Amato, M. R. (1973). Delayed matching and short-term memory in monkeys. In G. H. Bower (Ed.), The psychology of learning and motivation: Advances in research and theory (Vol. 7, pp. 227-269). New York: Academic Press.

Edhouse, W. V., W Wite, K. G. (1988). Sources of proactive interference in animal memory. Journal of Experimental Psychology: Animal Behavior Processes, 14, 56-70.

Franchina, J. J., \& Brown, T. S. (1971). Reward magnitude shift effects in rats with hippocampal lesions. Journal of Comparative \& Physiological Psychology, 76, 365-370.

Gaffan, E. A., Hansel, M. C., \& Smith, L. E. (1983). Does reward depletion influence spatial memory performance? Learning \& Motivation, 14, 58-74.

HoffMan, N., \& MAKI, W. S. (1986). Two sources of proactive interference in spatial working memory: Multiple effects of repeated trials on radial maze performance by rats. Animal Learning \& Behavior, 14, $65-72$

Hulse, S. H. (1978). Cognitive structure and serial pattern learning by animals. In S. H. Hulse, H. Fowler, \& W. K. Honig (Eds.), Cognitive processes in animal behavior (pp. 311-340). Hillsdale, NJ: Erlbaum.

Kraemer, P. J., Gilbert, M. E., \& InNis, N. K. (1983). The influence of cue type and configuration upon radial-maze performance in the rat. Animal Learning \& Behavior, 11, 373-380. 
Makı, W. S., Beatty, W. W., \& Clouse, B. A. (1984). Item and order information in spatial memory. Journal of Experimental Psychology: Animal Behavior Processes, 10, 437-452.

MaKI, W. S., HoffMan, N., FRITSCHE, B. M. (1986). Release from proactive interference by experimental amnesia: Electroconvulsive shock improves spatial memory in rats. Behavioral \& Neural Biology, 45, 300-318.

OLtoN, D. S. (1978). Characteristics of spatial memory. In S. H. Hulse, H. Fowler, \& W. K. Honig (Eds.), Cognitive processes in animal behavior (pp. 341-373). Hillsdale, NJ: Erlbaum.

Olton, D. S. (1979). Mazes, maps, and memory. American Psychologist, 34, 583-596.

Olton, D. S., Collison, C., \& Werz, M. A. (1977). Spatial memory and radial maze performance in rats. Learning \& Motivation, 8 , 289-314.

Olton, D. S., \& SAMUELSon, R. J. (1976). Remembrance of places past: Spatial memory in rats. Joumal of Experimental Psychology: Animal Behavior Processes, 2, 97-116.
Roberts, W. A., \& DALE, R. H. I. (1981). Remembrance of places lasts: Proactive inhibition and patterns of choice in rat spatial memory. Learning \& Motivation, 12, 261-281.

Santiago, H. C., \& Wright, A. A. (1984). Pigeon memory: Same/ different concept learning, serial probe recognition acquisition, and probe delay effects on the serial-position function. Journal of Experimental Psychology: Animal Behavior Processes, 4, 498-512.

Wright, A. A., Santiago, H. C., \& Sands, S. F. (1984). Monkey memory: Same/different concept learning, serial probe acquisition, and probe delay effects. Journal of Experimental Psychology: Animal Behavior Processes, 4, 513-529.

Wright, A. A., Urcuiol, P. J., \& SAnds, S. F. (1986). Proactive interference in animal memory. In D. F. Kendrick, M. E. Rilling, \& M. R. Denny (Eds.), Theories of animal memory (pp. 101-125). Hillsdale, NJ: Erlbaum.

(Manuscript received April 27, 1993; revision accepted for publication September 8, 1993.) 